\title{
Precursors, aftershocks, criticality and self-organized criticality
}

\author{
Y. Huang ${ }^{1}$, H. Saleur ${ }^{1}$, C. G. Sammis ${ }^{2}$, D. Sornette ${ }^{3,4}$ \\ 1 Department of Physics, University of Southern California, Los-Angeles, CA 90089-0484. \\ 2 Department of Earth Sciences, University of Southern California, Los-Angeles, CA 90089-0740. \\ ${ }^{3}$ Department of Earth and Space Sciences and Institute of Geophysics and Planetary Physics \\ University of California, Los Angeles, California 90095-1567 \\ ${ }^{4}$ Laboratoire de Physique de la Matière Condensée, CNRS URA 190 \\ Université des Sciences, B. P. 70, Parc Valrose, 06108 Nice Cedex 2, France
}

(May 13, 2021)

\begin{abstract}
We present a simple model of earthquakes on a pre-existing hierarchical fault network. The system self-organizes on long time scales in a stationary state with a power law GutenbergRichter distribution of earthquake sizes. The largest fault carries irregular great earthquakes preceded by precursors developing over long time scales and followed by aftershocks obeying an Omori's law. The cumulative energy released by precursors follows a time-to-failure power law with log-periodic structures, qualifying a large event as an effective dynamical (depinning) critical point. Down the hierarchy, smaller earthquakes exhibit the same phenomenology, albeit with increasing irregularities.
\end{abstract}

PACS numbers: 72.10.Fk, 73.40.Hm, 75.10.Fk.

Seismologists model dynamical rupture propagation with complex friction laws and barriers [1] and attempt to ascribe the earthquake complexity to nonlinear processes and/or heterogeneities. From a more global point of view, it has been suggested that earthquakes are somewhat similar to critical points [2], and can be addressed using tools of the renormalization group. A very broad, but quite ill-defined, perspective is also available with the concept of self-organized criticality [3,4]. These various points of view model different properties at different time scales; it is hard to see how they relate to each other, and whether they are part of a unique meaningful "theory of earthquakes". A closely related puzzle is whether criticality and self organized criticality are compatible.

The present work attempts to unify a significant fraction of this earthquake phenomenology, and to answer this puzzle: we define a simple model that exhibits the self-critical organization of the crust at large time scales, the critical nature of large earthquakes and the shorttime rupture dynamic properties.

We start with the following ingredients. (i) The faults are organized in a hierarchical geometrical structure [5.6. We do not address the problem of the construction of the fault patterns themselves which involves much larger times scale $\left(10^{5-6}\right.$ years $)$ compared to the time scales we describe $\left(10^{0-5}\right.$ years $)$. (ii) The tectonic plate is driven at a slow average uniform rate and we take into account its heterogeneities and the existence of relaxation processes by allowing for fluctuations in the local rate of loading. (iii) When a threshold is reached, a redistribution occurs on adjacent faults, with amplitude controlled by the size of the faults.

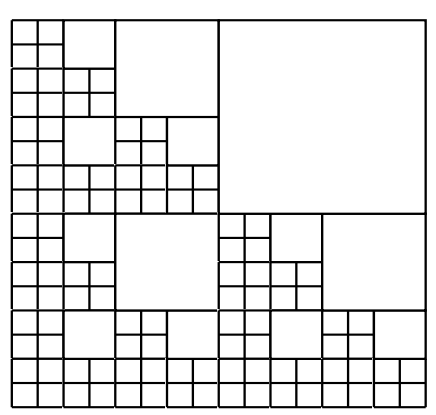

FIG. 1. The fractal cell structure.

Our model is an hybridization of the sandpile model [3] and of the fractal automaton [7]. As in [7], the cell sizes are arranged as a discrete fractal lattice. Each cell can be viewed as representing the region which is elastically unloaded when a fault fails. The fractal distribution of cell sizes then represents a fractal distribution of fault lengths [5]. The number of fractal generations does not appear to be a crucial parameter. Most simulations were carried out with 8 generations, but some runs with 12 generations did not exhibit major differences. We load the system by dropping particles at regular intervals (which we use as a clock) onto the grid at random sites. The addition of a particle is analogous to energy loading. The probability that a particle is added to a particular cell is proportional to the area $A$ of this cell. Although the exact cell (fault domain) to receive the next increment in stress is random, the entire grid is loaded uniformly at a uniform rate over the long-term. This represents the long-term uniform strain at the boundaries between moving tectonic plates. The short term random heterogeneity in loading represents heterogeneity in crustal structure or in upper mantle flow and the associated relaxation processes. Each cell becomes unstable when it contains $n \times 4 A$ particles, $n$ being a parameter. It then breaks and redistributes $4 A$ of its particles to its immediate neighbors. The number of particles redistributed to an adja- 
cent cell is proportional to the linear dimension of the cell. The $(n-1) \times 4 A$ particles that are not redistributed are considered as lost, like the particles which are redistributed outside the grid on the plate border. Such energy losses we call "cooling". Since the particles represent energy, the model assumes that a fault fails when the stored energy reaches a critical threshold. The key difference with [3] is that the energy must reach the critical level over the entire area of a cell before it is allowed to break. Due to the fractal structure, cells of widely different sizes are thus coupled together, mimicking the multi-scale interactions between faults.

The clock is defined by the particle drops. Cascades are triggered by the addition of a single additional particle, i.e. they occur instantaneously (a delay can also be introduced in the aftershock sequences, see below). At variance with the rules of [7] and in accord with the standard sandpile model [3], the size of an earthquake is determined by the size of the cascade, and is proportional to the total number of particles cascading. We thus identify the cascade as complexity in the mainshock, e.g. the linking of fault strands and segments or even the linking of adjacent faults such as occured in the 1994 Landers earthquake [8]. We define precursors as those regional cascades which precede a mainshock and the aftershocks as the cascades which follow it. We then use the time scale defined by the particle drops to explore the temporal structure of both the foreshock and aftershock sequences.

The cooling, i.e. the disappearence of particles during an event, represents a loss of stored elastic energy due to the earthquake. Consider for instance an elastic medium under constant applied shear strain, which suddenly undergoes a rupture in the form of a dislocation or a crack. The stress field is redistributed with enhancement at the crack tip as well as screening at some other places, while both the total shear stress and total elastic energy decrease. The amount of loss is a function of the nature of the rupture and of geometry. For instance, in the Griffith problem, a crack of length $2 c$ is introduced into a rod of length $L$ and section $\sim L^{2}$. Under constant strain, the relative energy loss $\frac{\delta E}{E}$ incurred by the introduction of the crack is $\frac{5 \alpha c^{2}}{L^{2}} /\left(1+\frac{5 \alpha c^{2}}{L^{2}}\right)$ [9] with $\alpha$ depending on the geometry and of the order of unity. If we take $c$ between $L / 4$ and $L / 2$, we get $\frac{\delta E}{E}$ anywhere between about $0.2-0.6$. This loss corresponds to friction on the fault plane, the creation of surface energy in the extensive crushing which occurs in the fault zone [6], and in the radiation of elastic waves 10 whose energy is ultimately lost as heat. Comparing with $\frac{\delta E}{E}=\frac{n-1}{n}$ in our model, we see that $n \approx 2$ is not unreasonable. We take this value for most of our simulations below. These large uncertainties in cooling are actually not critical in fact, provided $n$ is not too close to 1 , the results are largely independent of $n$. The choice $n=1$ of [7] leading to internal conservation (except at the boundaries) does not seem relevant for earthquakes.

As in [7], we identify an event cascading through the largest cell on the grid as a main shock for our system, i.e., the largest regional event. After a transient depending on the initial conditions, the system self-organizes in a stationary state with a power law Gutenberg-Richter distribution of earthquake sizes $P(E) d E \sim E^{-(1.8 \pm 0.1)} d E$. This can be called a self-organized critical state, measured by a statistics encompassing many times the largest time intervals between the largest earthquakes. We generated about 100 main shocks on an $8^{t h}$ order fractal structure by adding a total of about $4.6 \cdot 10^{7}$ particles to the system. The average number of time units (particle drops) between main shocks is $T=4.6 \cdot 10^{5}$, with fluctuations of this interval of the order of $10 \%$. This quasiperiodicity occurs because our main shocks are characteristic earthquakes [1] which completely control the energy redistribution at large scales. Their existence is not in contradiction with the self-organized critical state: they simply arise because of finite size effects.

For the purpose of comparison with real earthquakes, we choose our units of time so that $T=100$ years, i.e. roughly $10^{4}$ time units correspond to two years. For most main shocks, precursory built-up of activity is clearly visible, and lasts $1-2 \cdot 10^{5}$ time units, or about $20-40$ years. A decaying activity posterior to the main shocks is also visible with a lifetime of about $0.1-.2 \cdot 10^{5}$ time units, i.e. about $2-4$ years. Quite often, the interval between two successive main shocks will not be as quiet, but is interrupted by the breaking of one of the second largest cells. The time interval between such smaller shocks is much less regular than the time interval between main shocks.

We start by discussing aftershocks. The common wisdom holds that aftershocks involve a time delay between the application of the stress and the subsequent rupture. This delay presumably involves an intermediate relaxation time, whose effect could resemble that of diffusion or visco-elastic processes. In the present model, the spatial heterogeneity of the loading rate already reflects the existence of delay mechanisms. Inspired by the analysis in terms of critical phenomena (see below), we then plot the cumulative number of events as a function of time 12 starting from an initial date of about $10^{4}$ time units posterior to $t_{c}$ and going backwards in time. If Omori's Law $\frac{d n(t)}{d t} \propto \frac{1}{\left(t-t_{c}\right)^{p}}$ holds, this gives $n(t) \sim\left(t-t_{c}\right)^{1-p}$ (the theoretical divergence at long times for $p<1$ is truncated by the existence of background seismicity). Fits performed for 15 of our model aftershock sequences gave a distribution of the exponent $p$ centered around $p=.9$, with small fluctuations, $p \in[0.85,1.05]$, in good agreement with the exponent measured for earthquakes. These results are independent of the value of the dissipation parameter $n$, provided $n$ is not too close to 1 . As $n \rightarrow 1$, 
in particular when $n=1$, as in [7], the exponent $p$ is often near 1 , although it has large fluctuations, and can be found as low as $p=0.7$. Simulations on other fractals indicate that the exponent $p$ is also near unity for different geometry.

The model can be improved by the addition of a delay mechanism with a characteristic time $\tau$ for the avalanche associated with the main shock (this has no qualitative effect on the statistics of events, nor on the analysis of precursors). This had the drawback of introducing one additional parameter, but allows a more natural analysis of the data. Fortunately, results were found totally insensitive to the value of $\tau$ over a wide range $\left(\tau \leq 10^{4}\right)$ and in perfect agreement with Omori's law again.

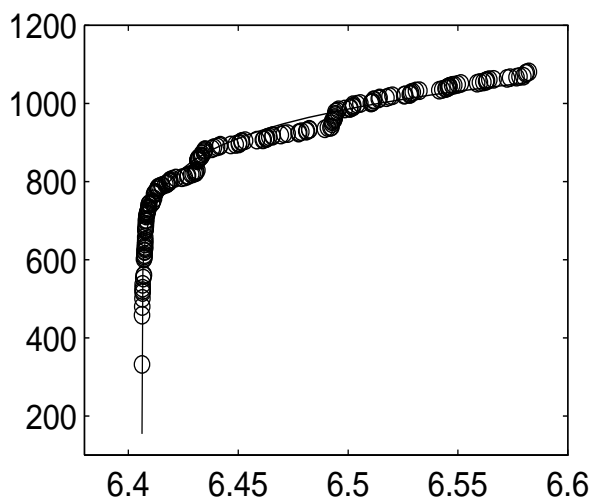

FIG. 2. Cumulative number of afttershocks as a function of time (in units of $10^{5}$ particle drops). The full line is a power law with $1-p=.12$.

We now turn to precursors. While virtually all large shallow earthquakes have easily recognizable aftershock sequences, the same can not be said for foreshocks. In fact, if foreshocks are defined to occur with the same time and space clustering as aftershocks, then most large events do not have a recognizable foreshock sequence 13] It is only when the time scale is extended to tens of years and the space to hundreds of kilometers that precursor sequences can be recognized $[14-16]$. We thus use the term "precursor" to distinguish the two definitions. We analyze data in the same spirit as in [16,17]: we plot the cumulative Benioff strain $\epsilon(t)$ (square root of the energy release in an event) as a function of time, starting back in time within a range $1-3 \cdot 10^{5}(20-60$ years $)$ prior to the main shock. We use a lower cut-off such as to exclude the crowd of small background events and test for various cut-off values. The conclusion is, in most cases, a reasonably clear evidence for a power law behavior, decorated by log periodic oscillations 16, 17.

$$
\epsilon(t)=A-B\left(t_{c}-t\right)^{m}\left[1+C \cos \omega \ln \left(t_{c}-t\right)\right] .
$$

The fit by this equation of the cumulative foreshock time series for the biggest main shock is shown in Figure 3.
This example is quite typical. Much better fits are sometimes obtained. Bad fits were rare but sometimes occured. The improvment of the $\chi^{2}$ when using (11) compared with a pure power law fit $(C=0)$ was always greater than two. The power law in (11) is the signature of a critical dynamical behavior, reminiscent of depinning transition.

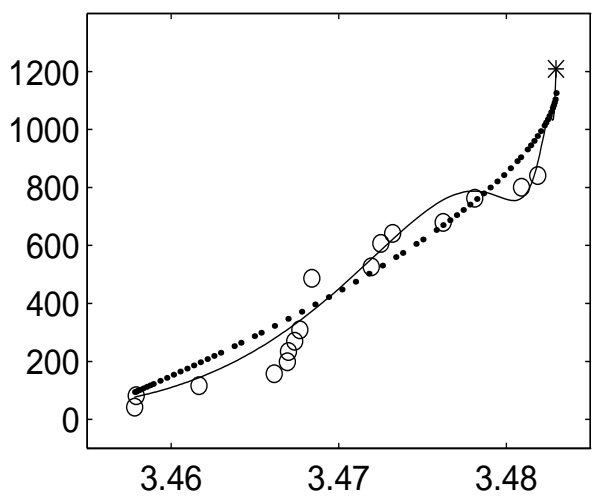

FIG. 3. Plot of Benioff strain before the biggest shock. The dotted line is power law behaviour, full line is power law decorated with log-periodic oscillations. The time is in units of $10^{7}$ particle drops.

The log-periodic correction in (11) reflects the discrete scale invariance (DSI) of the hierarchical fault network on which the events occur [17] (we observed similar oscillations decorating the Gutenberg-Richter law). Intriguingly, the amplitude $C$ of these oscillations is much bigger than for equilibrium statistical mechanics models [18]: the threshold dynamics in the earthquake model seems much more sensitive to DSI. We discuss below how the value of $\omega$ is related to our fractal structure.

Values of $m$ and $\omega$ fluctuate more than the exponent $p$ for aftershocks. In $80 \%$ of the cases however, $m$ was found in the interval $m \in[0.2,0.6]$, in good agreement with experimental data [16, 17. $\omega$ was found in the interval $\omega \in[6,12]$ corresponding to $1.7 \leq \lambda \leq 2.8$ where $\omega \equiv$ $2 \pi / \log \lambda$.

These results are not sensitive to the dissipation parameter $n$. Only the time scale is modified. In the extreme case of $n=1$ (without cooling), the seismic activity is much more random, and the rate of events looks much more constant between main shocks than it does with cooling: only about $10^{4}$ time units (2 years) before the main shock does the cumulative Benioff strain develop a power law behavior on the approach to the main shock, with properties that are then similar to the case $n=2$.

Beside the Benioff strain (11), we studied the correlation length $\xi$ defined as the maximum spreading distance of the cascades. To get more stable numerical results, we calculated its integrated value, which does exhibit a power law singularity described by 


$$
\begin{aligned}
& \xi \propto\left(t_{c}-t\right)^{-\nu_{1}}, t<t_{c}, \nu_{1} \in[0.6,0.8] \\
& \xi \propto\left(t-t_{c}\right)^{-\nu_{2}}, t>t_{c}, \nu_{2} \approx 1 .
\end{aligned}
$$

$\nu_{1}$ and $\nu_{2}$ have smaller fluctuations than the exponent $m$ of the Benioff strain. Observe that they are different on both sides of the critical point, a situation which is known to be possible in disordered systems in particular. The divergence of $\xi$ in (2) confirms the critical point picture. Moreover, the exponent provides a relation between DSI in time and DSI in space. From our fractal geometry, the latter is characterized by $x \rightarrow 2 x$. Substituting $\xi \rightarrow 2 \xi$ in (2) implies $\left|t-t_{c}\right| \rightarrow(\lambda)^{-1}\left|t-t_{c}\right|$ with $\lambda \in[2.4-3.2]$, i.e $\omega \in[5.4,7.2]$, in reasonable agreement with what we directly observed.

We now use the time-to-failure law (11) to try to forecast the main shock by fitting it to the "experimental" data up to a cutoff time prior to the main shock, as proposed in [16]. Overall, we find that $95 \%$ of the main shocks can be predicted with an uncertainty less than a year, four years in advance. This must be compared with the typical fluctuations of about 10 years of the time intervals between two main consecutive shocks. There are however cases where the predictability is much higher, and also extreme cases where the precursory activity is essentially nonexistent.

We also studied events occuring on the second largest cells. The analysis is slightly more complicated because two such events can be close in time but well-separated in space. We therefore restricted our attention to well isolated cases. The analysis of precursors and aftershocks gives similar results as for the foregoing events on largest cells. The only difference is that the time scales involved are shorter (roughly by a factor of 10), and the fluctuations in exponents somewhat bigger. This is presumably due to the fact that the relative size of the fluctuations of the "energy" field with respect to that of these earthquakes is larger for smaller events due to the influence of the earthquakes at the upper levels.

Our results thus indicate that reasonable intermediatetime earthquake prediction may be achievable, as proposed in 16,17. From the simulations, there is clear evidence that the predictabilty depends on the "temperature" of the system - the larger the loss of energy after a main shock, the better is the prediction of the next one. This is particularly clear for the first main shocks obtained when initiating the empty system, for which predictabilty is very high. The astonishing accuracy observed in [16] for the Loma Prieta example might thus be due to the existence, in that case, of a very "cool" seismic system.

In addition, we have demonstrated for the first time the possible coexistence of self-organized criticality and criticality. Up to now, they were considered as dual (mutually exclusive) modes of behavior: critical depinning occurs when the applied force reaches a critical value beyond which the system moves globally, while self-organized criticality needs a slow driving velocity and describes the jerky steady-state of the system. The critical nature of our large cascades emerges from the interplay between the long-range stress-stress correlations of the self-organized critical state and the hierarchical geometrical structure: a given level of the hierarchical rupture is like a critical point to all the lower levels, albeit with a finite size. The finite size effects are thus intrinsic to the process.

[1] A. Cochard and R. Madariaga R., Pure and Applied Geophysics 142, 419 (1994); J.M. Carlson, J.S. Langer and B.E. Shaw, Rev. Mod. Phys. 66, 657 (1994); Y. Benzion and J. Rice, J. Geophys. Res.100, 12959 (1995).

[2] C.J. Allègre, J.L. Le Mouel and A. Provost, Nature 297, 47 (1982); T.L. Chelidze, Phys. Earth Planet.Int. 28, 93 (1982); A.Sornette and D.Sornette, Tectonophysics 179, 327 (1990); A.G. Tumarkin and M.G. Shnirman, Computational seismology 25, 63 (1992).

[3] P. Bak P. and C. Tang, J.Geophys.Res.94, 15635 (1989).

[4] A.Sornette and D.Sornette, Europhys.Lett. 9, 197 (1989).

[5] G.C.P. King, Pageoph 124, 567 (1983); 123, 806 (1985).

[6] D.J. Andrews, J.Geophys.Res. 94, 9389 (1989).

[7] B. Barriere and D.L. Turcotte, Phys. Rev. E 49, 1151 (1994).

[8] Y.G. Li, J.E. Vidale, K. Aki, C.J. Marone et al., Science 265, 367 (1994).

[9] C.H. Scholz, The mechanics of earthquake and faulting (Cambridge University Press, Cambridge, UK, 1990).

[10] H. Kanamori, J. Mori, E. Hauksson, T.H. Heaton, L.K. Hutton and L.M. Jones, Bull. Seismol. Soc. Amer. 83, 330 (1993).

[11] D.P. Schwartz and K.J. Coopersmith, J.Geophys.Res. 89, 5681(1984)

[12] When computing this cumulative number, every breakdown in a cascade is counted as one event - this amounts to putting a delay of one time unit.

[13] D.C. Agnew and L.M. Jones, J. Geophys. Res. 96, 11959 (1991).

[14] L.R. Sykes and S. Jaumé, Nature 348, 595 (1990).

[15] L. Knopoff, T. Levshina, V.I. Keilis-Borok and C. Mattoni, J. Geophys. Res. 101, 5779 (1996).

[16] D. Sornette and C.G. Sammis, J.Phys.I France 5, 607 (1995)

[17] H. Saleur, C.G. Sammis and D. Sornette, J.Geophys.Res. 101, 17661 (1996); D.J. Varnes and C.G. Bufe, Geophys. J. Int. 124, 149 (1996).

[18] B. Derrida, C. Itzykson, J. M. Luck, Comm. Math. Phys. 94, 115 (1984). 